\title{
OPTICAL PROPERTIES AND PHOTOEMISSION OF MICRORELIEF SURFACES OF III-V SEMICONDUCTORS
}

\author{
N.L. DMitruk and S.V. Mamikin \\ Institute for Physics of Semiconductors, Ukrainian National Academy of Sciences \\ Kiev, Ukraine
}

The optical properties (infrared reflectance) and the photoemission current for the surface-barrier structures of metal-semiconductor type with microrelief interface have been investigated. The participation of surface plasmon polaritons in internal photoemission of the Au-GaAs Schottky barriers has been observed.

PACS numbers: 68.35 . Bs, 73.40.Ns, 78.30.Fs

Randomly rough (microrelief) surfaces prepared by wet chernical anisotropic (non-polishing) etching are especially interesting in physics of surfaces and interfaces. In this case we can create surface relief with certain geometric statistical parameters, without introducing a damage of the layer and with improved electronic properties such as minimal surface states concentration and surface recombination velocity.

Besides, microprofiling of the surface of a serniconductor by anisotropic chemical etching results in:

1) An increase in the absorption of light by the semiconductor ("trapping" of light);

2) An increase in the emission current, both the internal photoemission at a contact between a metal and a semiconductor and the field emission into a vacuum;

3) Enhancement of an electric field near the microscopic asperities;

4) Creation of the conditions for excitation of surface electromagnetic waves (surface polaritons) [1]. This causes enhancement of mixed electron-polariton phenomena which lead to a rise of electron (photoelectron) emission yield. The periodic and quasiperiodic surfaces or diffraction gratings made by interference photoetching method are of special interest. 
In this paper some of the above-mentioned physical phenomena have been illustrated by our experimental results on surface-barrier structures (SBS) of metal-semiconductor type.

The technology of the microrelief surface preparation has been developed for III-V semiconductors, GaAs and InP. It included preliminary cleaning of samples in organic solvents, removal of an imperfect layer, and chemical polishing in corresponding solution. Subsequent etching in concentrated acids $\mathrm{HNO}_{3}$ for $\mathrm{GaAs}$ and $\mathrm{H}_{2} \mathrm{SO}_{4}, \mathrm{HCl}$ for InP, respectively, produced microrelief surface $[2,3]$.

The microreliefs of anisotropically $\mathrm{HCl}$ etched surface of InP single crystals are quasiperiodical structures with periods distributed quasicontinuously in the range of tenths to dozens $\mu \mathrm{m}$. It was found that the etching of $n$ - and $p$-InP in concentrated $\mathrm{HCl}$ results in approximately parallel grooves. oriented along the $\langle 011\rangle$ and $\langle 01 \overline{1}\rangle$ directions on the $(100)$ and $(\overline{100)}$ surfaces, respectively. For the studied samples the groove cross-section is symmetric and its parameters (period, depth) approximately coincide for both sides (Fig. 1).

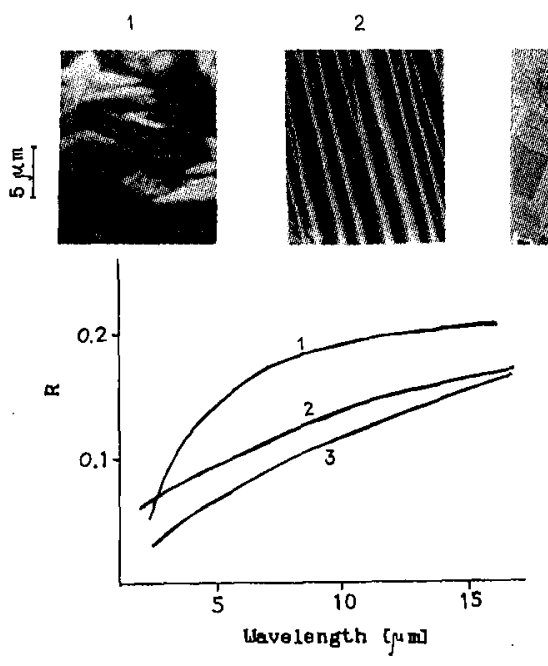

3
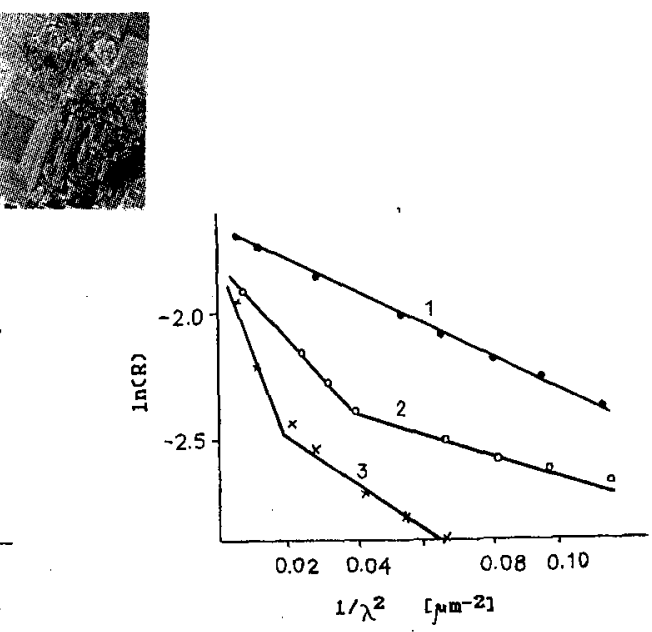

Fig. 1. The dependences of the reflectivity coefficient $R$ on $\lambda$ and $\ln (R)$ on $1 / \lambda^{2}$ for microrelief InP surfaces of different morphologies: curves $1-\mathrm{H}_{2} \mathrm{SO}_{4}, 2-\mathrm{HCl}$; $3-\mathrm{H}_{2} \mathrm{SO}_{4}: \mathrm{HCl}=1: 1$. Upper inset shows SEM photos of the anisotropically etched surfaces.

The specular reflection spectra are smooth curves (Fig. 2), decaying monotonically towards short wavelengths.

It is well known that the near normal specular reflection from Gaussian-like surface $R(\lambda)$ has the following form:

$$
R=\bar{r} \exp \left(-\pi^{2} h^{2} / \lambda^{2}\right),
$$

where $\bar{r}$ is the angle-average Fresnel reflection coefficient near the incidence angle, $h$ is the root-mean-square height. In that case the value of $\ln (R)$ has a linear dependence on $1 / \lambda^{2}$ and its slope depends on $h^{2}$. Figure 1 confirms this fact: a straight 


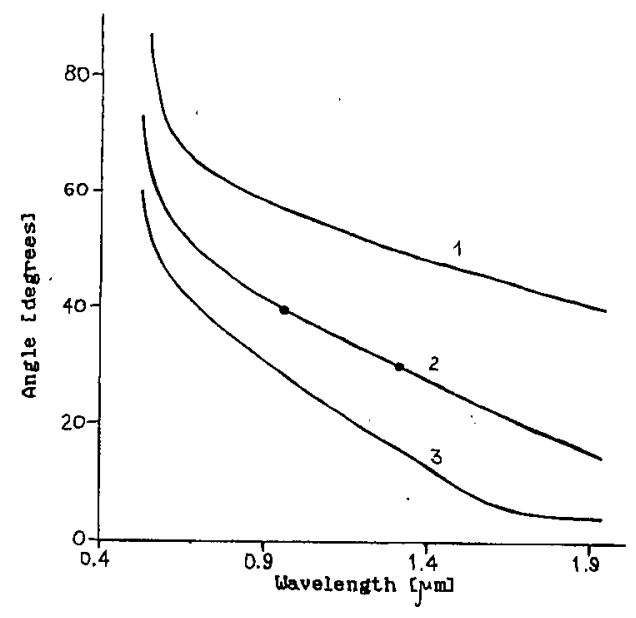

Fig. 2. The dependences of light-incidence angles at which surface polariton is excited on wavelength for three diffraction gratings with periods: $1-5.5 \mu \mathrm{m} ; 2-2.6 \mu \mathrm{m}$; $3-1.8 \mu \mathrm{m}$.

line is observed for the stars-containing relief and a broken line for pyramids- and grooves-containing reliefs of InP. The latter suggests that two components are present in the reliefs. Root-mean-square roughness $(\delta)$ correlates with those obtained from relief measurements. The equation $h=4 \delta$ is valid for the isotropic reliefs and according to the theory indicates the Gaussian distribution of roughness. On the other hand, this equation is invalid for anisotropic (2D) surfaces. In any case the specular reflection is significantly reduced by the surface relief in the spectral range $\lambda \ll \delta, \sigma ; \sigma$ is the autocorrelation length. The specular reflection coefficient decreases to about $3 \%$ in the visible spectrum range. Therefore, the experimentally observed photocurrent of the metal-serniconductor barrier structures with a microrelief interface is by 3-50 times greater than in the case of a flat interface [4].

An investigation of the spectra of the photoemission current of $\mathrm{Au}-\mathrm{GaAs}$ diodes, carried out at $T=100 \div 300 \mathrm{~K}$ and $h \nu=0.7 \div 1.3 \mathrm{eV}$, showed that near the fundarnental absorption edge of $\operatorname{GaAs}\left(\varphi_{\mathrm{b}}<h .>E_{\mathrm{g}}\right)$ a Fowler photocurrent $i_{\mathrm{ph}}$ was observed for flat structures. The surface microrelief increases several times the photoemission current in comparison with a flat surface and it leads to the shift of the spectral characteristics toward long wavelengths. At low temperatures $(T<200 \mathrm{~K})$ and in the range $h \nu<\varphi_{\mathrm{b}}$ a photocurrent of the opposite polarity was observed.

The anomalous photocurrent at $h \nu<\varphi_{\mathrm{b}}$ appears due to the photoexcitation of electrons from the valence band to unoccupied surface states followed by their tunnelling into the metal [5].

We observed for the first time the participation of the surface plasmon polaritons of the metal film in the photoernission current of Schottky barrier. According to the theory (see, for example [1]) the surface plasmon polaritons spectrum 
consists of two branches, the so-called fast and slow rnodes, which are localized on the interface of metal-air and metal-semiconductor, respectively.

Because these modes are non-radiative their excitation is possible only on periodic or quasiperiodic microrelief surfaces (diffraction gratings). The upper (fast) polariton branch of the air-Au-GaAs system coincides with the spectral region of the internal photoemission on the $\mathrm{Au}-\mathrm{GaAs}$ interface. In Fig. 2 the dependences of the incidence angle of light $\theta$ on the wavelength are shown for three different diffraction gratings. Experimentally the excitation of surface polariton modes is accompanied by the decreasing mirror reflected component of light and the increasing photoemission current. In Fig. 3, the angle dependences of the photoemission current for diffraction grating Au-GaAs at two frequencies of light are shown. Non-monotonous behaviour of these curves in the case of $p$-polarized light and smooth ones for $s$-polarized light indicate the excitation of surface plasmon polaritons. The angular position of these maxima $i_{\mathrm{ph}}(\theta)$ are in good agreement with theoretical dispersion equation of fast surface plasmon polaritons of $\mathrm{Au}-\mathrm{GaAs}$ system (see points on curves of Fig. 2).

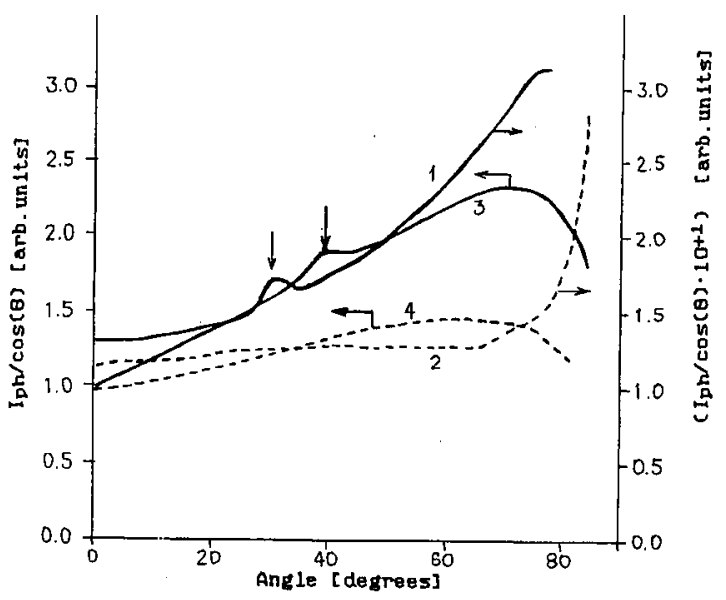

Fig. 3. Experimental angle dependences of photoemission current for frequencies $h \nu=1.0 \mathrm{eV}(1,2)$ and $h \nu=1.3 \mathrm{eV}(3,4)$ at $p-(1,3)$ and $s-(2,4)$ polarization of light, respectively.

We conclude with the following remarks. The regimes which provide definite morphology and statistical parameters of the surface rricrorelief, have been developed and analysed for both the GaAs and $\mathrm{InP}$ anisotropic etching and interference photo-etching. The Gaussian distribution of surface roughness is provided by the study of the reflection spectra of light within a wide wavelength range and by the profilometry. The photoemission current of microrelief surface barrier structures of metal-semiconductor type is several times greater in corriparison with the case of a flat interface. It occurs due to a sharp decrease in light reflection and improvement of surface electronic structure which take place due to anisotropic 
etching. The participation of the surface plasmon polaritons of the metal film has been observed in the photoemission current of Schottky barrier with the periodic interface.

\section{Acknowledgments}

The work was partially supported by Ukrainian Committee for Scientific Research and Technology.

\section{References}

[1] N.L. Dmitruk, V.G. Litovchenko, V.L. Strishevskyi, Surface Polaritons in Semiconductors and Dielectrics, Naukova Dumka, Kiev 1989, p. 375 (in Russian).

[2] N.L. Dmitruk, N.V. Kotova, E.V. Pidlisnyi, T.R. Barlas, Phys. Solid State 35, 8 (1993).

[3] N.L. Dmitruk, T.R. Barlas, E.V. Basiuk, Surf. Sci. 293, 107 (1993).

[4] O.Yu. Borkovskaya, N.L. Dmitruk, O.N. Mishchuk, Sov. Phys. Semicond. 25, 294 (1991).

[5] N.L. Dmitruk, V.G. Litovchenko, O.N. Mischchuk, Sov. Phys. Solid State 34, 1418 (1992). 